\title{
Réhabilitation prothétique implanto-prothétique sur un greffon de fibula après exérèse carcinologique chez un enfant. A propos d'un cas
}

\section{Prosthetic implant restoration in pediatric post-carcinologic fibula graft. Report of a case}

HERVÉ LÉON*, JEAN LOZZA*, JEAN-CLAUDE VERNIĖRES*, CHRISTIAN DELESTAN*, PEDRO MONTOYA**, PHILIPPE LAPEYRIE*

\section{RÉSUMÉ}

Cet article présente le cas d'un enfant âgé de dix ans, traité pour un synovialosarcome biphasique mandibulaire et ayant subi une hémimandibulectomie gauche. La reconstruction de l'arc mandibulaire est réalisée à l'aide d'une greffe microvascularisée de fibula. La solution implantaire est choisie pour permettre la réhabilitation esthétique et fonctionnelle. Le pronostic est favorable et le jeune patient a totalement retrouvé l'intégrité de son système manducateur. (Med Buccale Chir Buccale 2005; 11:17-22).

médecine buccale chirurgie buccale

VOL. $11, \mathrm{~N}^{\circ} 1$ 2005

page 17

\section{SUMMARY}

This paper is about a 10 years old child, treated for a biphasic synovialosarcoma of the mandible and who has gone throught the removal of the left hemimandible. The restoration of the mandible arch has been carried out with a fibula microvascular graft. The implant solution has been chosen to allow esthetic and functional rehabilitation. The prognosis was favourable and the young patient has totally recovered his manducatory system. (Med Buccale Chir Buccale 2005; $11: 17-22)$.

key words: Dental implants, children, synovialosarcoma, fibula bone graft. 
médecine buccale chirurgie buccale

VOL. $11, \mathrm{~N}^{\circ} 1$ 2005 page 18
Un enfant de dix ans a présenté un synovialosarcome biphasique mandibulaire développé dans la région de la première prémolaire mandibulaire gauche avec envahissement de la loge submandibulaire. Après échec de la chimiothérapie, le traitement de cette tumeur maligne a consisté en une large chirurgie d'exérèse, complétée par une radiothérapie [1]

Dans un deuxième temps la reconstruction de la continuité mandibulaire a été assurée par un transfert libre de fibula: cette indication est motivée par l'importance de la perte de substance et par la possibilité d'adaptation à la forme de l'hémimandibule réséquée [2,3]. Pour rétablir un équilibre occlusal optimum et pour stimuler le greffon, le choix de la réhabilitation prothétique s'est porté sur une prothèse partielle adjointe, stabilisée par des implants dentaires placés dans le greffon, dans un deuxième temps opératoire [4-6].

\section{OBSERVATION}

Un enfant âgé de 10 ans est vu en consultation en août 1997 pour une mobilité de la première prémolaire mandibulaire gauche (34), une masse submandibulaire gauche immobile, fixée aux plans profonds, associée à une asthénie et une anorexie importante (amaigrissement de $9 \mathrm{~kg}$ en un mois) n'ayant pas répondu aux traitements anti-infectieux et anti-inflammatoires. En octobre 1997, une première biopsie, confirmée par une seconde, révèle que la tumeur est constituée par un synovialosarcome [7-12]

Par ailleurs, les aires ganglionnaires sont libres, le myélogramme est normal et il n'y a pas d'extension méningée.

L'examen tomodensitométrique montre une masse, à grand axe transversal, mesurant $40 \times 50 \times 30 \mathrm{~mm}$, située entre les espaces parapharyngés et le plancher buccal, et associée à des zones lytiques du corpus mandibulaire gauche, classée T2b No Mo, Stade II.

Un avis spécialisé est pris auprès de l'Institut Gustave Roussy (I.G.R.). Le traitement est inclus dans le protocole NMT 95 qui comporte pour un stade II trois cures d' I.V.A. :

- Ifosfamide ${ }^{\circledR} 3$ g.m ${ }^{-2}$ à $J_{1}$ et $J_{2}$.

- Vincristine ${ }^{\circledR} 1,5 \mathrm{mg} \cdot \mathrm{m}^{-2}$ à $\mathrm{J}_{1}$.

- Actinomycine $D^{\circledR} 1,5$ mg.m. $m_{-2}$ à $J_{1}$.
Le bilan d'évaluation effectué après cette chimiothérapie, le 3 décembre 1997, ne montre pas d'augmentation de volume de la masse tumorale, mais il y a une extension de la lyse osseuse avec atteinte des deux corticales de la mandibule et une perte spontanée des dents dans le quadrant III.

Une chimiothérapie de deuxième ligne par C.E.V est instaurée:

- Carboplatine ${ }^{\circledR} 500 \mathrm{mg} \cdot \mathrm{m}^{-2}$ à $\mathrm{J}_{1}$.

- Epirubicine ${ }^{\circledR} 75 \mathrm{mg} \cdot \mathrm{m}^{-2}$ à $\mathrm{J}_{1}$ et $\mathrm{J}_{2}$.

- Oncovin ${ }^{\circledR 1,5}$ mg.m² à $\mathrm{J}_{1}$.

Trois cures seront réalisées, le 18 décembre 1997, le 12 janvier 1998, le 2 février 1998, suivies par une exérèse chirurgicale de la tumeur pratiquée le 13 février 1998 dans le Service de Chirurgie Infantile du C.H.U. de Montpellier.

La pièce de résection mandibulaire a emporté la totalité du corpus et du ramus mandibulaires gauches. Elle mesure $9 \mathrm{~cm}$ de long, sur laquelle adhère une tumeur nodulaire blanchâtre de $60 \mathrm{x}$ $45 \times 40 \mathrm{~mm}$ sans foyer de nécrose et ayant infiltré les plans osseux.

L'examen anatomopathologique confirme le diagnostic de synovialosarcome de type biphasique grade I, à prédominance épithéliale. Une radiothérapie complémentaire par électronthérapie est démarrée le 16 mars 1998 au Centre anti-cancéreux de Val d'Aurelle, en prévoyant une marge de sécurité au niveau cutané.

Les suites opératoires et les différents contrôles sont bons. Cependant, étant donné le jeune âge du patient et pour prévenir les inévitables séquelles esthétiques et fonctionnelles qu'entraîne une hémimandibulectomie pratiquée en période de croissance, on réalise dans le même service une reconstruction par greffe microvascularisée de fibula en mai 1998 [13].

Après consolidation du greffon et récupération d'une ouverture buccale symétrique, liée entre autre au contour harmonieux de la face, il est décidé d'appareiller la zone édentée pour rétablir la fonction [14-16]. Du fait du décalage important des appuis osseux dans le sens vertical et de l'épaisseur du lambeau muqueux ne pouvant pas supporter une prothèse partielle adjointe, il est envisagé d'utiliser des implants dentaires pour supporter la prothèse [17-19]. II est donc procédé à la pose de quatre implants dentaires en titane $\left(\right.$ Biotech $\left.^{\circledR}\right)$ vissés, 


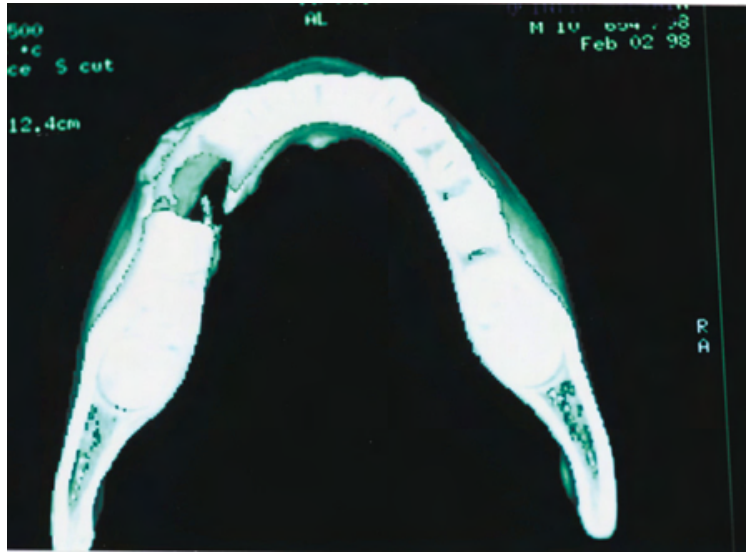

Figure 1: scanner de la mandibule avant l'intervention chirurgicale.

Mandible of before surgical operation.

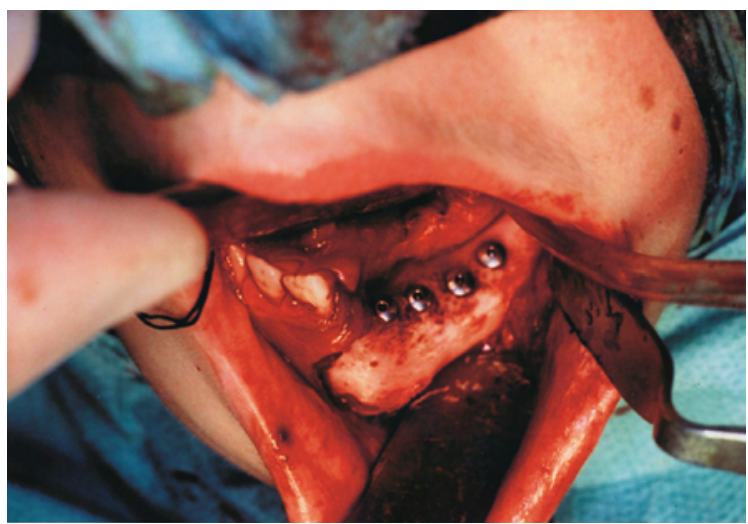

Figure 3: vue per-opératoire des implants dentaires dans le greffon de fibula.

Per-operating view of dental implants in fibula graft.

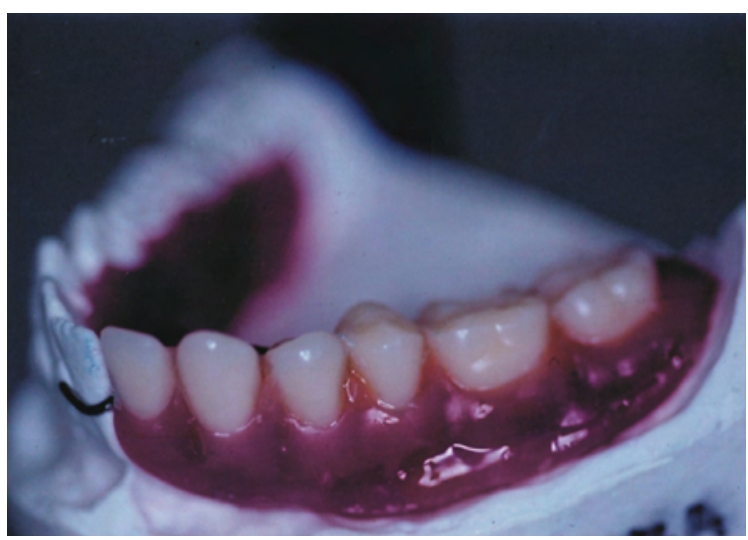

Figure 5: réalisation de la prothèse partielle adjointe. Removable partial prosthesis in plaster model.

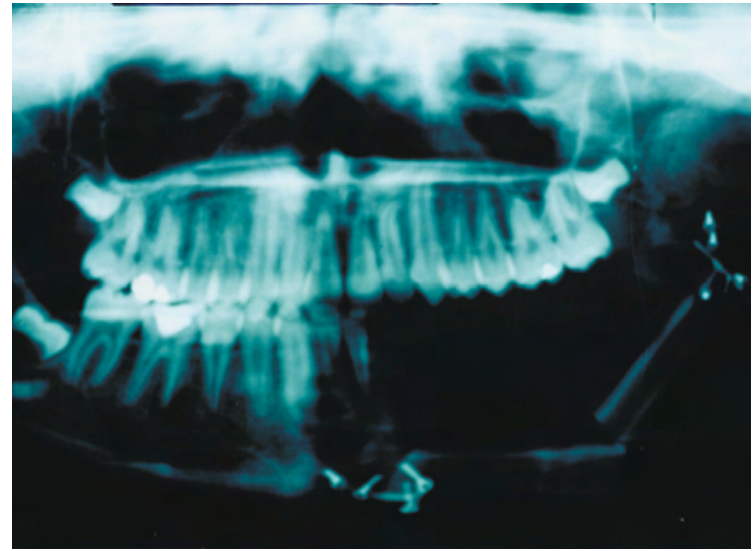

Figure 2: orthopantomogramme après la greffe de fibula.

Orthopantomography after fibula graft.

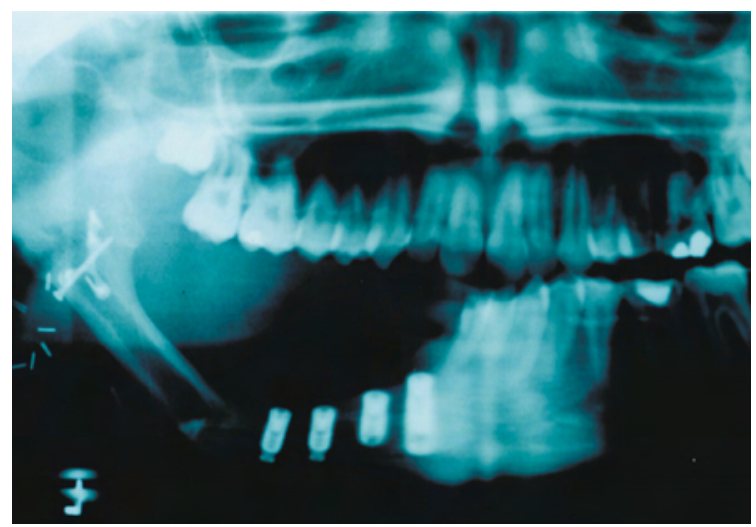

médecine

buccale

chirurgie

buccale

VOL. $11, N^{\circ} 1$

2005

page 19

Figure 4: orthopantomogramme après la pose des implants dentaires dans le greffon de fibula.

Orthopantomography after dental implants placementin fibula graft.

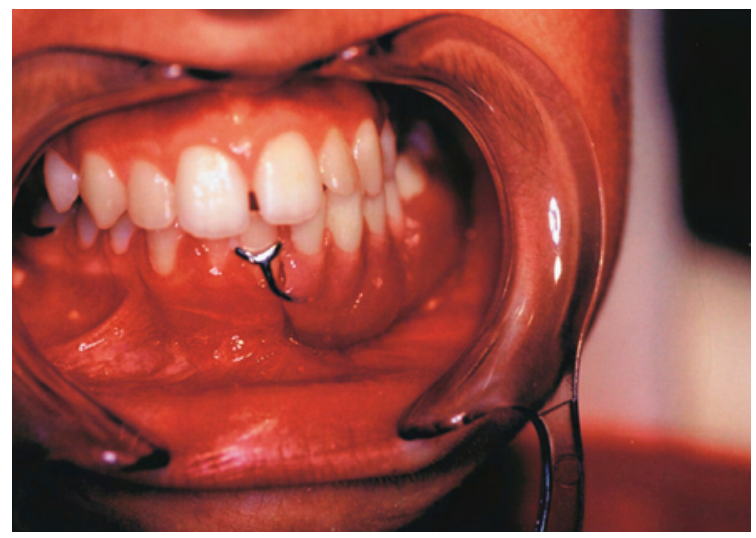

Figure 6: essayage de la prothèse partielle adjointe. Fitting of removable partial prosthesis. 
médecine

buccale

chirurgie

buccale

VOL. $11, \mathrm{~N}^{\circ} 1$ 2005

page 20 anti-rotationnels, de diamètre $3,9 \mathrm{~mm}$, de longueur $10 \mathrm{~mm}$ et $16 \mathrm{~mm}$ pour le plus antérieur. Pendant l'intervention le matériel d'ostéosynthèse et les vis sont déposés [20]. Les zones à implanter et le parallélisme des implants sont repérés par un guide chirurgical préparé à l'avance sur une maquette de reconstruction prothétique par un wax-up. L'emplacement des implants est localisé au moyen d'une fraise boule de gros diamètre. On fore les avant-trous dans la position et la direction voulue avec un forêt jetable de 1,4 mm de diamètre, à la longueur prédéterminée sur le scanner, en se servant du guide chirurgical [21-23]. On fore ensuite les fûts implantaires, distants chacun d'environ 3 à 4 mm, à l'emplacement approximatif des dents 32, 34, 35 et 36 . Cette opération se réalise sous irrigation interne avec du sérum physiologique réfrigéré, à l'aide de forêts de diamètres croissants ( $2 \mathrm{~mm}, 3 \mathrm{~mm}$ et $3,5 \mathrm{~mm}$ ). Le contrôle peropératoire est fait au moyen de jauges de profondeur qui peuvent également contrôler le parallélisme. Les implants auto-taraudants sont vissés manuellement au moyen de la clef à cliquet, puis protégés par une vis de couverture. On procède ensuite à la fermeture du site opératoire en deux plans. Le 4 février 2000, après contrôle radiographique de l'ostéointégration des implants, on procède à l'operculisation et à la pose de quatre piliers implantaires droits.

Dans les suites, une réaction inflammatoire bénigne de type bourgeon charnu, sans signe histologique de malignité, impose une retouche chirurgicale de la muqueuse le 24 mars 2000, afin de dégager les piliers prothétiques implantaires qui étaient partiellement réenfouis. Afin de solliciter mécaniquement l'os greffé et pour permettre la croissance du greffon, la prothèse est en appui uniquement sur les piliers implantaires. Une prothèse partielle adjointe est mise en place le 24 mai 2000 en prenant en compte des paramètres particuliers, comme l'absence d'appui muqueux sur le greffon, une occlusion non traumatogène et la possibilité de retouches ultérieures [24].

Les différents contrôles cliniques et radiologiques effectués depuis cette date sont satisfaisants et la réhabilitation prothético-implantaire remplit son rôle fonctionnel et esthétique. Sur les clichés orthopantomographiques de contrôle, on a mesuré une légère croissance du greffon en hauteur et consta- ter une harmonisation de sa forme justifiant à posteriori la décision d'une réhabilitation prothétique précoce.

\section{COMMENTAIRES}

Le synovialosarcome est une tumeur maligne mésenchymateuse que l'on rencontre très rarement dans la région cervicocépahlique. II est connu pour son caractère agressif et son risque élevé de métastases, notamment pulmonaires. II survient essentiellement chez l'adolescent, touchant les extrémités, en particulier des membres inférieurs. Sur le plan clinique, il se présente comme une masse indolore, augmentant progressivement de volume sur plusieurs mois, jusqu'à l'apparition de signes d'appel comme une dyspnée, une dysphagie ou une dysphonie, signant une compression pharyngolaryngée, ou bien comme une masse cervicale ou endobuccale ne répondant pas aux traitements anti-infectieux et anti-inflammatoires.

Dans le cas de cet enfant, le diagnostic histopathologique était celui d'un synovialosarcome biphasique de grade I, à prédominance épithéliale. Le traitement multimodal de cette tumeur permet un meilleur contrôle tumoral local et de métastases à distances. Cependant, la chirurgie d'exérèse reste le facteur déterminant pour le traitement et le suivi à long terme.

La reconstruction des pertes de substance étendues de la mandibule par greffon libre de fibula est une intervention bien codifiée [25, 26], qui permet un bon remodelage du contour inférieur de la face [27, 28]. Malgré le jeune âge du patient, il a été décidé de procéder à une reconstruction prothétique sur des implants dentaires [29]. Placés dans le greffon, ils permettent d'assurer, d'une part un équilibre occlusal stable, et d'autre part de stimuler le transplant qui peut bénéficier du potentiel de croissance actif dans la zone condylienne, à condition qu'il y ait une stimulation biomécanique.

La première difficulté rencontrée a été celle de réaliser la chirurgie implantaire sous anesthésie générale car il a été difficile d'obtenir la collaboration de cet enfant aux lourds antécédents thérapeutiques. Pour la partie prothétique (empreintes, occlusion, essayages, retouches...), sa compliance a été 
obtenue seulement après de longues discussions et en présence d'un membre de sa famille.

Un guide radiologique et un guide chirurgical ont été utilisés pour déterminer l'emplacement favorable des implants. Mais il y avait une seconde difficulté : le support osseux était totalement différent de l'infrastructure mandibulaire habituelle, et l'environnement muqueux particulièrement défavorable avec une gencive attachée totalement absente, remplacée par un lambeau musculo-muqueux très épais, non adhérent au greffon sous-jacent [30, 31]. Ceci a conduit à éviter un contact trop étroit entre la prothèse et le revêtement muqueux, aboutissant ainsi à une prothèse partielle adjointe implanto-portée.

Un autre problème s'est posé lors de la phase de réhabilitation prothétique: la hauteur des piliers implantaires vissés fournis par le fabricant étant insuffisante; ils ont été remplacés par des vis en titane habituellement utilisées comme transfert d'empreinte. Ceci a permis de compenser le décalage vertical dû à la différence de hauteur entre le corpus mandibulaire et le greffon de fibula.

\section{RÉFÉRENCES}

1 - MiLleR MJ. Osseous tissue engineering in oncologic surgery. Semin Surg Oncol 2000; 19: 294-301.

2 - Captier G, Montoya P, Duche T, Le Barazer M, BigorRe M, MARgueritTE G. Synovialosarcome de la mandibule chez l'enfant. A propos d'une observation. Rev Stomatol Chir Maxillofac 1999; 100 : 187-91.

3 - HAUGHEY BH, FREDRICKSON JM, LERRICK AJ, SCLAROFF A, GAY WD. Fibular and iliac crest osteomuscular free flap reconstruction of oral cavity. Laryngoscope 1994; 104 : 1305-13.

4 - Beckers A, Schenck C, Klesper B, Koebke J. Comparative densitometric study of iliac crest and scapula bone in relation to osseous integrated dental implants in microvascular mandibular reconstruction. J Craniomaxillofac Surg 1998; 26: 75-83.

5 - GuRlek A, MILLER MJ, JACOB RF, LIVELY JA, SCHUSTERMAN MA. Functional results of dental restoration with osseointegrated implants after mandible reconstruction. Plast Reconstr Surg 1998; 101 : 650-5 (discussion 656-9).

6 - BAHR W. Maxillary augmentation with a microvascularly anastomosed fibula: a preliminary report. Int J Oral Maxillofac Implants 1996; 11: 719-27.

7 - Gullane P, Kraus D, Weber R. Soft tissue sarcoma. Head Neck 2002 ; 24 : 296-300.
En raison de l'utilisation de piliers prothétiques présentant un rapport défavorable en fonction de la longueur des implants, il était dangereux pour la pérennité de la reconstruction, d'envisager une prothèse conjointe scellée ou vissée. C'est ce qui a motivé le choix pour une prothèse adjointe partielle à appui implantaire.

La croissance et l'évolution d'un greffon chez un patient âgé de douze ans reste encore hypothétique [32].

La conception de la prothèse ainsi que le relief occlusal des dents artificielles évitant toute force tangentielle nocive pour les implants, le moyen de rétention utilisé est représenté par deux crochets simples sur les dents restantes, comme pour une prothèse classique.

Lors des différents contrôles radiographiques d'usage - les radiographies ayant été réalisées avec le même appareil et avec le même technicien [33] - on a pu constater un remodelage fonctionnel du greffon avec harmonisation de l'angle goniaque, et une croissance en épaisseur de trois à quatre millimètres en un an.

8 - CIHAK RA, LYDIATT WM, LYDIATT DD, BRIDGE JA. Synovial sarcoma of the head and neck: chromosomal translation (X; 18) as a diagnostic aid. Head Neck 1997 ; 19 : 549-53.

9 - BuKACHEVSKy RP, PINCUS RL, SheChtMAN FG, SARTI E, CHODOSH P. Synovial sarcoma of the head and neck. Head Neck 1992; 14 : 44-8.

10 - Pruszczynski M, MAnNI JJ, Smedts F. Endolaryngeal synovial sarcoma: case report with immunohistochemical studies. Head Neck 1989; 11 : 76-80.

11 - NADIG SK, LOVE MH, BROOKER DS, HALL SJ. Synovial sarcoma in the retropharyngeal space. J Laryngol Otol 2002; 116: 224-6.

12 - ALBERTY J, DOCKHORN-DWORNICZAK B. Monophasic synovial sarcoma of the neck in an 8-year-old girl resembling a thyroglossal duct cyst. Int J Pediatr Otorhinolaryngol $2002 ; 63: 61-5$.

13 - MYOUNG H, KIM YY, HeO MS, LeE SS, CHOI SC, KIM MJ. Comparative radiologic study of bone density and cortical thickness of donor bone used in mandibular reconstruction. Oral Surg Oral Med Oral Pathol Oral Radiol Endod 2001; 92 : 23-9.

14 - Olstad OA, SKJELBRED P, LYBERG T. Reconstruction of the jaw and oral cavity with free vascularized grafts. lidsskr Nor Laegeforen 1996 ; 116: 2431-5. médecine buccale chirurgie buccale

VOL. $11, \mathrm{~N}^{\circ} 1$ 2005

page 21 
15 - Barber HD, Seckinger RJ, Hayden RE, Weinstein GS. Evaluation of osseointegration of endosseous implants in radiated, vascularized fibula flaps to the mandible: a pilot study. J Oral and Maxillofac Surg 1995 ; 53 : 6404 (discussion 644-5).

16 - Keller EE, Tolman DE, ZUCK SL, ECKERT SE. Mandibular endosseous implants and autogenous bone grafting in irradiated tissue: a 10-year retrospective study. Int J Oral Maxillofac Implants 1997 ; 12 : 800-13.

17 - SUMI Y, HASEGAWA T, MIYAISHI O, UEDA M. Interface analysis of titanium implants in a human vascularized fibula bone graft. J Oral Maxillofac Surg 2001; 59 : 213-6.

18 - Chiapasco M, Abati S, Ramundo G, Rossi A, Romeo E, VoGEL G. Behavior of implants in bone grafts or free flaps after tumor resection. Clin Oral Implants Res 2000; 11 (1) : 66-75.

19 - Nagy K, Borbely L, Kavocs A, Vajdovich I, Mari A. Implant-prosthetic rehabilitaton after segmental mandibulectomy and bone grafting. J Long Term Eff Med Implants 1999; 9: 185-91.

20 - Huault M, Laroche C, LeVy J, Laxenaire $A$, ROUCAYROL AM, SchefFer P. Epithélioma cuniculatum. A propos d'un cas gingival antérieur avec extension

médecine buccale chirurgie buccale

VOL. $11, \mathrm{~N}^{\circ} 1$ 2005

page 22 osseuse mandibulaire symphisaire et reconstruction par lambeau oséo-cutané fibulaire et pose d'implants. Rev Stomatol Chir Maxillofac 1998; 99: 143-8.

21 - Moscoso JF, Keller J, Genden E, Weinberg H, BILLER HF, BUCHBINDER D, URKEN ML. Vascularized bone flaps in oromandibular reconstruction. A comparative anatomic study of bone stock from various donor sites to assess suitability for enosseous dental implants. Arch Otolaryngol Head Neck Surg 1994; 120 : 36-43.

22 - HuRny JM, ZLototlow IM, PIRO JD, LENChEWSKI E. Osseointegrated implants in microvascular fibula free flap reconstructed mandibles. J Prosthet Dent 1993; 70: 443-6.

23 - ZLOTOLOW IM, HURNY JM, PIRO JD, LENCHEWSKI E, HIDALGO DA. Osteointegrated implants and functional prosthetic rehabilitation in microvascular fibula free flap reconstructed mandibles. Am J Surg 1992; 164 : 677-81.

24 - JACOB RF, KING GE. Partial denture framework design for bone-grafted mandibles restored with osseointegrated implants. J Prosthodont 1995; 4 : 6-10. 